\title{
Dendritic excitability modulates dendritic information processing in a Purkinje cell model
}

\author{
Allan D. Coop ${ }^{1}$, Hugo Cornelis ${ }^{2}$ and Fidel Santamaria ${ }^{3 *}$ \\ 1 Department of Epidemiology and Biostatistics, University of Texas Health Science Center at San Antonio, San Antonio, TX, USA \\ 2 Research Imaging Centre, University of Texas Health Science Center at San Antonio, San Antonio, TX, USA \\ 3 Biology Department and Neurosciences Institute, University of Texas at San Antonio, San Antonio, TX, USA
}

\section{Edited by:}

Peter Dayan, University College

London, UK

Reviewed by:

Mickey London, University of

Hertfordshire, UK

Volker Steuber, University of

Hertfordshire, UK

*Correspondence:

Fidel Santamaria, Department of Biology, University of Texas at San

Antonio, San Antonio, TX 78249, USA.

e-mail: fidel.santamaria@utsa.edu
Using an electrophysiological compartmental model of a Purkinje cell we quantified the contribution of individual active dendritic currents to processing of synaptic activity from granule cells. We used mutual information as a measure to quantify the information from the total excitatory input current $\left(I_{\text {Glu }}\right)$ encoded in each dendritic current. In this context, each active current was considered an information channel. Our analyses showed that most of the information was encoded by the calcium $\left(I_{\mathrm{CaP}}\right)$ and calcium activated potassium $\left(I_{\mathrm{Kc}}\right)$ currents. Mutual information between $I_{\text {Glu }}$ and $I_{\text {CaP }}$ and $I_{\mathrm{Kc}}$ was sensitive to different levels of excitatory and inhibitory synaptic activity that, at the same time, resulted in the same firing rate at the soma. Since dendritic excitability could be a mechanism to regulate information processing in neurons we quantified the changes in mutual information between $I_{\mathrm{Glu}}$ and all Purkinje cell currents as a function of the density of dendritic $\mathrm{Ca}\left(g_{\mathrm{CaP}}\right)$ and $\mathrm{Kca}\left(g_{\mathrm{Kc}}\right)$ conductances. We extended our analysis to determine the window of temporal integration of $I_{\mathrm{Glu}}$ by $I_{\mathrm{CaP}}$ and $I_{\mathrm{KC}}$ as a function of channel density and synaptic activity. The window of information integration has a stronger dependence on increasing values of $g_{\mathrm{Kc}}$ than on $g_{\mathrm{CaP}}$, but at high levels of synaptic stimulation information integration is reduced to a few milliseconds. Overall, our results show that different dendritic conductances differentially encode synaptic activity and that dendritic excitability and the level of synaptic activity regulate the flow of information in dendrites.

Keywords: information theory, dendritic conductances, synaptic plasticity, dendritic computation, cerebellum, firing rate, modulatory synapses, compartmental modeling

\section{INTRODUCTION}

The contribution of the passive dendritic properties of neurons on processing of synaptic activity is well established (Rall, 1962a,b; Marr, 1969; Pellionisz and Szentagothai, 1973, 1974); however, possibly all central nervous system neurons express voltage sensitive dendritic conductances (Llinas and Yarom, 1981; Hirst and McLachlan, 1986; Hockberger et al., 1989; Huguenard et al., 1989; Masukawa et al., 1991; Segev and London, 1999). Active conductances can carry more current than passive conductances and, thus, have a stronger contribution to the processing and transfer of synaptic activity in dendritic trees (Gollo et al., 2009). Furthermore, the multiple types of conductances found in dendrites could differentially filter synaptic activity depending on its temporal and spatial distribution (Poirazi and Mel, 2001; Migliore and Shepherd, 2002). Unfortunately, the lack of experimental access to determine the distribution and kinetics of active membrane conductances in dendrites has not allowed a thorough study of their influence on processing of synaptic activity.

In order to track the transformation of synaptic activity, and thus obtain a representation of the transfer function of the dendrite, it is necessary to determine how synaptic activity is distributed over different dendritic currents. In many cases the variability observed in dendritic currents is driven by synaptic activity and can be regulated by interactions among conductances. Quantifying the amount of variability due to synaptic activity or due to internal interactions can be done using with statistical tools derived from information theory (Shannon, 1948; Shannon and Weaver, 1949; Rieke, 1997). Particularly, determining mutual information (Foffani and Priori, 2007; Maffezzoli et al., 2008; Quian Quiroga and Panzeri, 2009) between synaptic activity and specific dendritic currents could result in a quantitative method to measure the contribution of individual dendritic currents on synaptic processing (Stemmler and Koch, 1999; London et al., 2002). Furthermore, the calculation of the entropy, conditional entropy and mutual information can help elucidate how much variability observed in the dendritic currents is due to the nature of the synaptic input or due to intrinsic properties of the dendrite.

We present the results of calculating mutual information between the net excitatory input and different dendritic currents using a detailed biophysical model of a Purkinje cell (De Schutter and Bower, 1994a,b). The model was stimulated with randomly distributed excitatory and inhibitory synapses that resembled granule cell and interneuron activity. We found that the calcium $(\mathrm{CaP})$ and a calcium activated potassium $(\mathrm{Kc})$ currents carried the largest amount of information. While the firing rate of the Purkinje cell remained constant for different combinations of synaptic input, mutual information was sensitive to such changes, thus disambiguating synaptic activity in dendrites. Our results suggest that dendritic excitability modulated by $\mathrm{CaP}$ and Kc channels is most effective in regulating the mutual information, and 
thus any possible processing of that information. Conceptually, our approach treats each dendritic conductance as an information channel that carries a certain amount of information depending on each conductance density. Since plasticity of dendritic excitability might be an important component of general learning and memory mechanisms in neurons (Xu and Kang, 2005; Komendantov and Ascoli, 2009), our results provide a method to quantify the effects of such changes on synaptic information processing in individual dendritic conductances.

\section{MATERIALS AND METHODS PURKINJE CELL MODEL}

We used a previously published Purkinje cell model (De Schutter and Bower, 1994b,c) with updated synaptic kinetics (Figure 1A). Please refer to the cited work for kinetic details of each conductance. In brief, there is one excitatory synaptic contact made with each dendritic spine and 1,695 inhibitory GABAa-type synaptic contacts distributed at random across the dendrites. The model contains the following dendritic conductances (Figure 1B): two types of Ca channels, a P-type, CaP (Llinas et al., 1989) and a T-type, CaT (Kaneda et al., 1990); two types of Ca-activated $\mathrm{K}^{+}$channels, a BK-type (Latorre et al., 1989) referred to here as Kc and a K2-type, K2 (Gruol et al., 1991); and a persistent $\mathrm{K}^{+}$channel or Km-type (Yamada et al., 1989). The soma had two types of sodium channels, a fast current, $\mathrm{NaF}$ (Hirano and Hagiwara, 1989) and a slow persistent current, NaP (French et al., 1990); one type of calcium current T-type; and four types of potassium channels, anomalous rectifier, Ih (Spain et al., 1987); delayed rectifier, Kdr (Yamada et al., 1989); persistent potassium, Km; and an A-type potassium channel, KA (Hirano and Hagiwara, 1989). In depth analysis of the model can be found elsewhere (De Schutter and Bower, 1994a,b,c; Jaeger et al., 1997; Gundappa-Sulur et al., 1999; Santamaria et al., 2002, 2007; Santamaria and Bower, 2005; De Schutter and Steuber, 2009). The updated synaptic properties are described in Santamaria et al. (2007).

\section{SIMULATIONS}

The simulations consisted in randomly activating all the excitatory and inhibitory synapses at constant Poisson firing rates. As explained in the Results we used four different combinations of

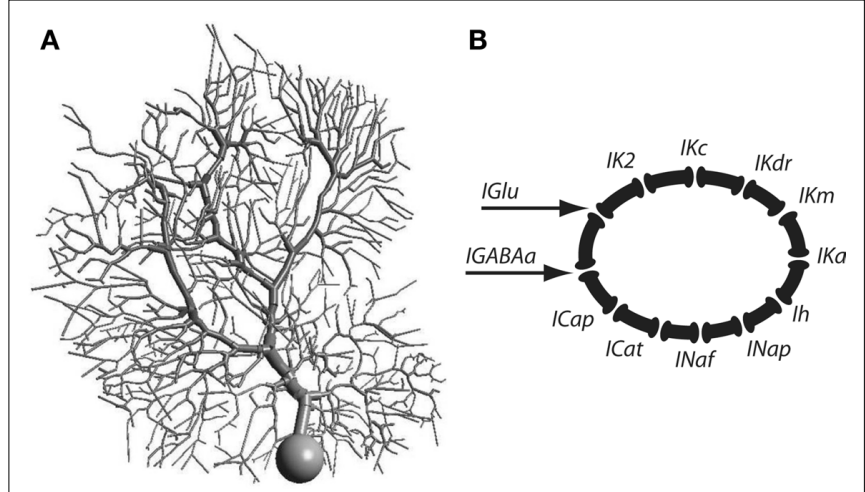

FIGURE 1 | (A) Morphology of the Purkinje cell model. (B) Schematic representation of the channel types incorporated into the Purkinje cell model. excitatory and inhibitory synaptic activity that resulted in the same firing rate at the soma of the Purkinje cell. We ran simulations for up to $400 \mathrm{~s}$ saving the value of all dendritic and synaptic currents every $100 \mu$ s. In order to avoid initial condition effects the first $5 \mathrm{~s}$ of all traces were not used for the analysis. Simulations were run with a pre-release version of the new GENESIS 3 software $^{1}$. Since each single simulation required about $25 \mathrm{~h}$ to complete we used a high performance computing cluster operated by the Computational Biology Initiative at UTSA ${ }^{2}$.

\section{INITIAL ANALYSIS}

In order to simplify the analysis we monitored the total value of the synaptic or dendritic currents. This approximation to the state of dendritic currents has allowed us to analyze this model in detail in the past with testable experimental predictions (Jaeger and Bower, 1999; Santamaria et al., 2007). We also chose to use the total excitatory current because then the results of our study could be mapped to dynamic current clamp experiments (see Discussion). We also ran our analysis under two other conditions. In the first one we added all currents over all the dendrites except the thick smooth dendrites (primary). The second condition was adding all the current exclusively in the spiny dendrites (tertiary), without the primary and secondary. The results were practically the same as the ones reported for the summed currents over the entire dendrite, indicating that all the integration is done at the level of tertiary dendrites.

For the purposes of comparing the changes due to background activity we normalized the value of all currents from 1-100 and binned the data in 1000 equally spaced bins. All the analyses described in this paper were performed with the normalized current values.

Initial characterization of currents was done by calculating the histograms under all the different combinations of synaptic activity. Further analysis consisted in calculating the crosscorrelation between the excitatory synaptic input $\left(I_{\mathrm{Glu}}\right)$ and dendritic currents.

\section{INFORMATION THEORY}

Since we wanted to characterize the amount of activity in dendritic currents due to synaptic stimulation we used information theory measurements. This process required calculating the entropy $(H)$ of each current, the conditional entropy between each dendritic current and $I_{\mathrm{Glu}}$, and finally the mutual information $(I)$.

The entropy was calculated as

$H(x)=\sum_{i} p\left(x_{i}\right) \log _{2} p\left(x_{i}\right)$

where $p$ is the probability of seeing value $x_{i}$.

The conditional entropy is a number that describes the variability of a signal given that the value of a second signal is known. This is a useful measurement when trying to understand the variability of the dendritic currents as a function of the synaptic current. We calculated the conditional entropy as

$H(y \mid x)=\sum_{j} \sum_{i} p\left(x_{i}, y_{j}\right) \log _{2} p\left(y_{j} \mid x_{i}\right)$

${ }^{1}$ http://www.genesis-sim.org/

${ }^{2}$ http://www.cbi.utsa.edu 
In our case, $x$ is the input signal $\left(I_{\text {Glu }}\right)$ and $y$ any of the dendritic currents. Conditional probability distributions matrices were calculated based on the binned traces. The conditional probability distributions did not result in distributions that allocated values to every matrix entry. This lack of probability space coverage was due to the biophysical restriction of the particular conductances. Coupling and long time constants also contributed to having areas in the probability density matrix that were densely populated while other remained empty.

Finally, the mutual information was calculated with eq. 3:

$I(y \mid x)=H(y)-H(y \mid x)$

The mutual information is usually expressed in bits and gives the capacity of a neuron to discriminate between different stimuli that are part of the stimulus, in this case $I_{\mathrm{Glu}}$. For example, if the mutual information is equal to 5 bits, this means that a particular dendritic current is able to distinguish $2^{5}$ or 32 different stimuli.

It is well known that the value of $I$ can be biased due to the binning process and finite size of the data being analyzed (Panzeri et al., 2007). We used a recently developed toolbox in Matlab (Natick, MA, USA) that allows the accurate calculation of the different information measurements and compensation for potential biases (Magri et al., 2009). Independently of using such compensations we determined that a 400 -s simulation was long enough by plotting the value of $I$ for any pair of $I_{\mathrm{Glu}}$ and dendritic current as a function of the time window of observation from 5-400 s. This analysis showed that after $300 \mathrm{~s}$ the value of $I$, without bias compensation, reached an asymptotic value (Figure $\mathbf{S 1}$ in Supplementary Material). In any case, the value of $I$ can be biased if the joint probability distribution of the two traces being analyzed, for example $I_{\mathrm{Glu}}$ and $I_{\mathrm{CaP}}$, is scattered and does not fill out the joint probability space, in our case a matrix of $1000 \times 1000$ entries. The ratio $N / m$ has been shown to determine the strength of such a bias, where $N$ is the number of non-zero entries in the joint probability distribution and $m$ number of non-zero entries of probability distribution of the stimulus. If $\mathrm{N} / \mathrm{m}$ is less than 1 then the value obtained from calculating the mutual information is biased. As shown in the supplementary materials all our simulations had an $\mathrm{N} / \mathrm{m}>1$.

\section{RESULTS}

The objective of our work was to quantify the contribution of dendritic excitability on information processing in dendrites. For this purpose we used an available Purkinje cell model since this type of cell has extensive dendritic conductances and this particular model has been well validated with experimental data (Jaeger and Bower, 1994, 1999; Jaeger et al., 1997; Santamaria et al., 2007).

\section{CALCIUM AND CALCIUM ACTIVATED POTASSIUM CHANNELS CARRY MOST OF THE MUTUAL INFORMATION IN THE PURKINJE CELL DENDRITE}

Information in Purkinje cells flows from synapses, to dendrites, to soma (Santamaria et al., 2007). As opposed to cortical pyramidal cells, Purkinje cells have no backpropagating action potentials that carry somatic activity into the dendrites (Vetter et al., 2001). Thus, Purkinje cells are ideal to quantify the incremental contribution of dendritic channels to information processing. Since excitatory synapses from parallel fibers to Purkinje cells carry the activity from outside the cerebellum (Bower, 2002) we assumed this stimulus to be the input signal. We characterized the input signal as the total excitatory current $\left(I_{\mathrm{Glu}}\right)$ because experimental techniques, such as dynamic current clamp, could be used to test modeling predictions based on this assumption (Suter and Jaeger, 2004). Purkinje cells in vivo receive excitatory synaptic activity from parallel fiber and inhibitory activity from interneurons; we simulated these types of inputs with random input firing rates drawn from a Poissonian distribution. Firing rates at the soma can be the result of multiple combinations of excitation and inhibition in the dendrite (Santamaria et al., 2002; Santamaria and Bower, 2005), for that reason we selected various pairs of uncorrelated background excitatory and inhibitory activity that evoked similar firing rates at the soma. Using different combinations of excitatory and inhibitory input with the same somatic firing rate allowed us to determine information processing changes in dendrites due to different levels of synaptic activity. The background input firing rates spanned a wide range of rates $12-56 \mathrm{~Hz}$ and $0.5-2.0 \mathrm{~Hz}$ for excitation and inhibition, respectively (Santamaria and Bower, 2005).

We initially quantified the effects of the random excitatory input on the histogram of values of each dendritic current. For the different combinations of background excitatory and inhibitory input we found that while the histogram of $I_{\text {Glu }}$ remained practically unchanged (Figure 2A) the shape of the distribution of the $\mathrm{CaP}\left(I_{\mathrm{CaP}}\right.$, Figure 2B $)$ and Kc currents $\left(I_{\mathrm{Kc}}\right.$, Figure $\left.2 \mathrm{C}\right)$ varied. All other dendritic currents $\left(I_{\mathrm{CaT}}, I_{\mathrm{Km}}\right.$, and $\left.I_{\mathrm{K} 2}\right)$ did not show changes in the shape of their distribution as a function of background levels of synaptic stimulation (Figures 2D-F). We did not analyze $I_{\mathrm{NaF}}, I_{\mathrm{Kdr}}, I_{\mathrm{A}}, I_{\mathrm{NaP}}$ and $I_{\mathrm{h}}$ because they are only present in the soma and the thick proximal dendrite. The mean firing rate $\left(F_{M}\right)$, calculated as the number of spikes across the entire simulation divided by the simulation time (400 s) shows that the different combinations of synaptic activity had little influence $(90.3 \pm 2.4$ spikes/s), Figure $2 G$, similarly the distributions arising from calculating the inter-spike interval histograms for all combinations of synaptic input (Figure $2 \mathbf{H}$ ) differ in less than $1 \%$ in the value of their expected value and standard deviation.

The analysis presented in Figure 2 suggests that most of the changes in background levels of synaptic activity affect the variability of $I_{\mathrm{CaP}}$ and $I_{\mathrm{Kc}}$. Without synaptic activity the Purkinje cell model is quiescent, thus, the source of the variability observed in the dendritic currents comes from synaptic activity and from interactions among channels. In order to quantify the amount of variability due to $I_{\text {Glu }}$ we made use of information theory (Victor, 2000; Nemenman et al., 2004; Paninski et al., 2004; Kennel et al., 2005). We initially calculated the entropy of $I_{\mathrm{Glu}}, I_{\mathrm{CaP}}$ and $I_{\mathrm{Kc}}$ (Figure 3A). In order to quantify the causal relationship between synaptic activity and dendritic currents we performed the information analysis with a small time lag. Thus, the conditional entropy and mutual information were calculated between the dendritic currents at time $t$ and the glutamate input at a time $t-\Delta t$, we assumed $\Delta t$ to be $1 \mathrm{~ms}$. The analyses show that the entropy of dendritic currents depends on the combination of background activity. The entropy of $I_{\mathrm{CaP}}$ increases and reaches the same magnitude as the entropy of $I_{\mathrm{Glu}}$ as a function of the frequency of the background synaptic activity. Note that all of our calculations were biased corrected using the Panzeri and Treves method (Magri et al., 2009). The conditional entropy quantifies the uncertainty in the value of a variable given the known value of another. Calculating the conditional entropy 

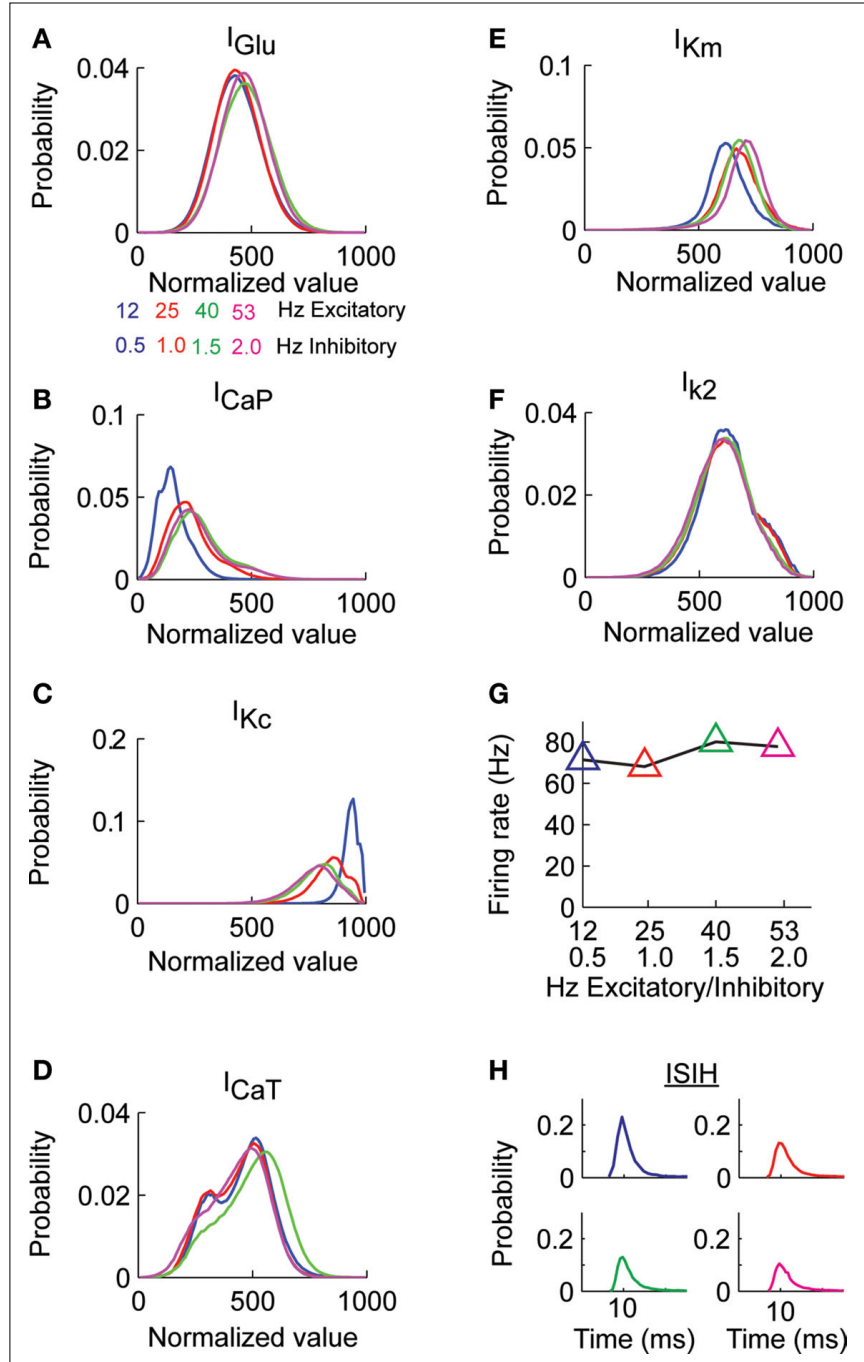

FIGURE 2 | Probability distribution of synaptic and dendritic currents. (A) The Purkinje cell model was stimulated with pairs of excitatory and inhibitory synaptic activity. The total excitatory synaptic current $\left(I_{\text {Glu }}\right)$ remained practically constant for all the combinations of excitatory and inhibitory activity (activity in $\mathrm{Hz}$ ). (B) Probability distribution of the $I_{\mathrm{CaP}}$ in response to the different combinations of excitatory and inhibitory activity in (A). (C) Probability distribution of $I_{\mathrm{KC}}$ for the same simulations in (B). (D-F) The probability distribution of the other dendritic currents remained practically independent of level of synaptic activity. (G) The average firing rate at the soma remains constant for all combination of synaptic activity. (H) The Purkinje cell inter-spike distributions for each combination of synaptic activity in (A) have the same mean and standard deviation.

of $I_{\mathrm{CaP}}$ and $I_{\mathrm{Kc}}$ with respect to $I_{\mathrm{Glu}}$ shows that the variability in the currents is high (Figure 3B), suggesting that most of the activity observed is due to intrinsic activity. However, the value of mutual information between $I_{\mathrm{CaP}}$ and $I_{\mathrm{Kc}}$ with respect to $I_{\mathrm{Glu}}$ shows an increase in information as a function of level of background level of stimulation (Figure 3C). The value of mutual information for the other calcium channel $\left(I_{\mathrm{CaT}}\right)$ was larger (Figure S2 in Supplementary Material) than for $I_{\mathrm{CaP}}$ but since must of the current flows through $I_{\mathrm{CaP}}$ (Santamaria et al., 2002) we only analyzed this channel (see Discussion). Overall, the analysis presented in

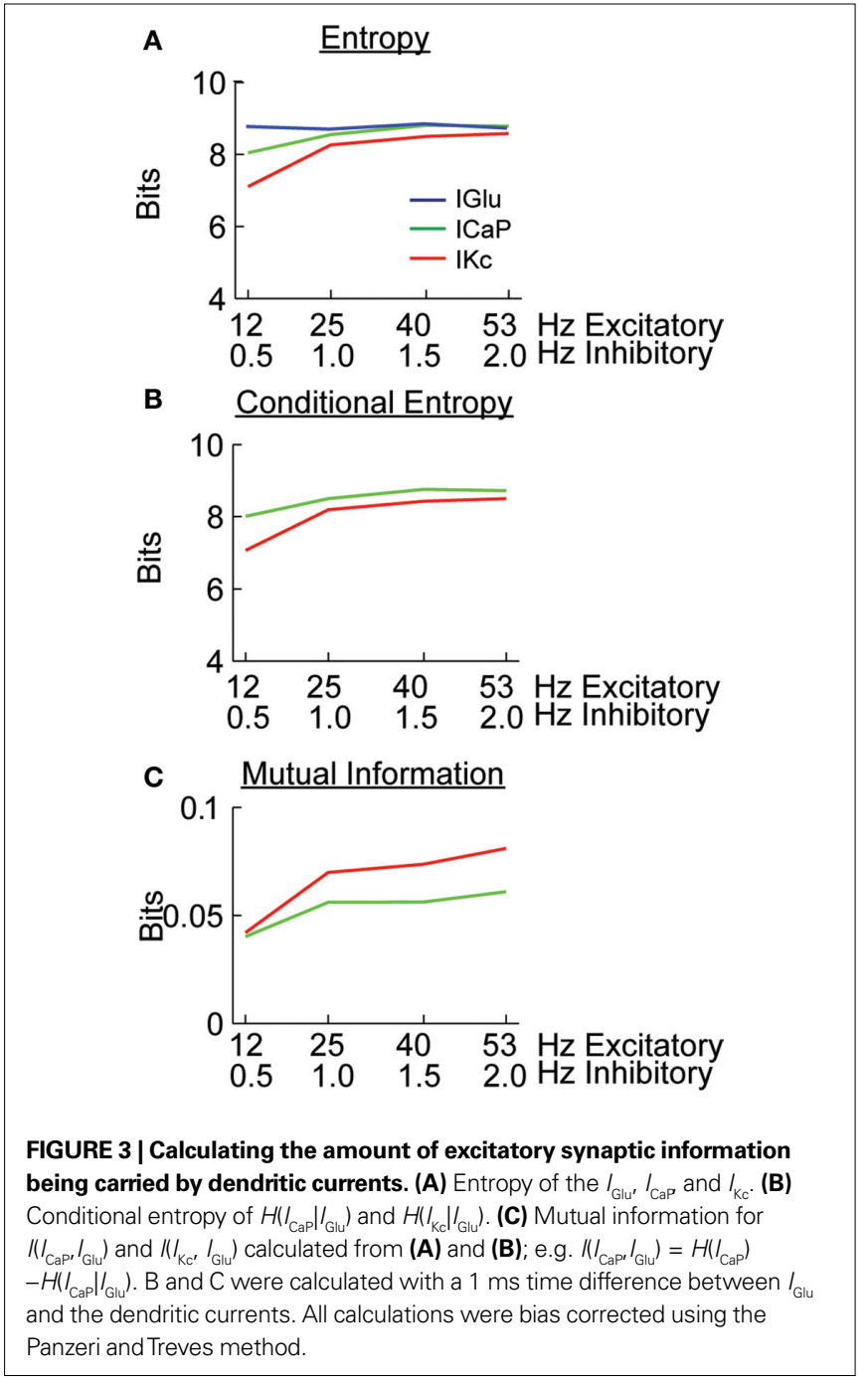

Figures 2 and 3; Figure S2 in Supplementary Material show that most dendritic currents, except for $\mathrm{CaP}$ and $\mathrm{K}_{\mathrm{C}}$, are not sensitive to changes in background synaptic activity. The mutual information of Kc with respect to $I_{\mathrm{Glu}}$ is larger than for the same measurement done with $\mathrm{CaP}$. However, the ratio of mutual information of these two currents changes as a function of the level of synaptic activity. More interestingly, this differential encoding of the background excitatory information can take place while the somatic firing rate remains constant.

\section{TEMPORAL INTEGRATION REGULATED BY SYNAPTIC ACTIVITY AND DENDRITIC EXCITABILITY}

The time constants of activation and inactivation of dendritic conductances imply that information content at one point in time is influenced by past synaptic activity. We characterized these time dependent changes by calculating the cross-correlation of $I_{G l u}$ with $I_{\mathrm{CaP}}$ and $I_{\mathrm{Kc}}$ for all different combinations of excitatory and inhibitory input (Figure 4). This analysis shows that as the excitatory input frequency increases the value of $I_{\mathrm{CaP}}$ (green) becomes more tightly coupled with $I_{\text {Glu }}$ (blue, auto-correlation), while the coupling of the $I_{\mathrm{Cap}}$ and $I_{\mathrm{Kc}}$ also increases as we have previously reported using 
phase plane analysis (Santamaria et al., 2002). The amplitude of lag 0 is indicative of the amount of correlation with previous activity. The half-time auto-correlation time between $I_{\mathrm{Glu}}-I_{\mathrm{CaP}}$ extracted from these plots ranges between 3-70 ms.

We determined how the value of mutual information between $I_{\mathrm{Glu}}$ and $I_{\mathrm{CaP}}$ and $I_{\mathrm{Kc}}$ varied as an effect of previous activity. We calculated the mutual information between $I_{\mathrm{CaP}}$ and $I_{\mathrm{Kc}}$ with $I_{\mathrm{Glu}}$ for lag times up to $1 \mathrm{~s}$. Similar to what we did in Figure 3, at a given time lag of $\Delta t$ we computed the mutual information of the value of $I_{\mathrm{CaP}}$ or $I_{\mathrm{Kc}}$ at time $t$ with the value of $I_{\mathrm{Glu}}$ at time $t-\Delta t$. This analysis shows that the mutual information between $I_{\mathrm{CaP}}$ and $I_{\mathrm{Glu}}$ decays as a function of time lag (Figure 5A). This decay is due to the leaky nature of the conductance and membrane system. However, as opposed to the cross-correlation analysis the mutual information only shows one secondary peak at around $20 \mathrm{~ms}$. Similarly, the time lag analysis of $I_{\mathrm{Glu}}$ and $I_{\mathrm{Kc}}$ show an initial decay of the mutual information and a peak at around $20 \mathrm{~ms}$ (Figure 5B). Channel kinetics and level of synaptic activity generate strong correlations at lag 0 which reflect the average level of activity in
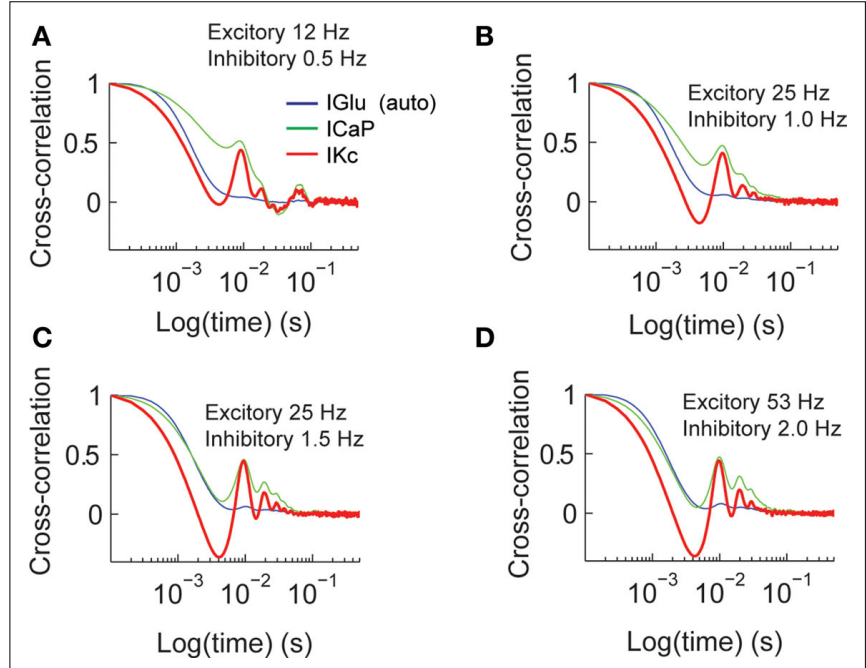

FIGURE 4 | Cross-correlation analysis of total synaptic excitatory input and dendritic currents. The figures show the auto-correlation of the synaptic current ( $I_{\mathrm{G} \mid \mathrm{u}^{\prime}}$ blue), and the cross-correlation of $I_{\mathrm{Glu}}$ with $I_{\mathrm{CaP}}$ (green), and $I_{\mathrm{KC}}$ (red). We repeated this analysis for all the combinations of synaptic activity (A-D). the dendrite, while the second peak is the mutual information in each channel from the instantaneous value of $I_{\mathrm{Glu}}$. As is known from functional and anatomical studies of the cerebellar cortex, excitatory inputs to Purkinje cells are divided in two groups. The first group comes from parallel fibers, which are being studied in this work; the second group is the ascending segment synapses (Llinas, 1982). The ascending segment synapses deliver a more synchronous input that forces the Purkinje cell to fire robustly (Santamaria et al., 2007). The information analysis presented in Figure 5 suggests that paired stimulus stimulation from ascending segment synapses would then be influenced in a window of around $20 \mathrm{~ms}$ by previous activity. This prediction is consistent with our previous analysis of ascending segment processing in the Purkinje cell model (Santamaria and Bower, 2005).

\section{DENDRITIC EXCITABILITY MODULATES INFORMATION CONTENT IN THE DENDRITE}

Plastic changes in the expression of active conductances affect the signal processing properties of dendritic trees (Segev and London, 1999; Stuart and Hausser, 2001). Each dendritic channel contributes to the encoding and transformation of synaptic input depending on channel kinetics and expression. Thus, we decided to quantify the dependence of the value of mutual information between $I_{\mathrm{Glu}}$ with $I_{\mathrm{CaP}}$ and $I_{\mathrm{Kc}}$ as a function of dendritic channel density.

We varied the excitability of the dendrite by changing the conductance of the $\mathrm{CaP}\left(g_{\mathrm{CaP}}\right)$ or $\mathrm{Kc}\left(g_{\mathrm{Kc}}\right)$. The value of $g_{\mathrm{CaP}}$ was varied from $25-70 \mathrm{~S} / \mathrm{m}^{2}$, while keeping $g_{\mathrm{Kc}}$ at its control value $\left(800 \mathrm{~S} / \mathrm{m}^{2}\right)$, and $g_{\mathrm{Kc}}$ ranged from $550-1000 \mathrm{~S} / \mathrm{m}^{2}$, while keeping $g_{\mathrm{CaP}}$ at the original value of $45 \mathrm{~S} / \mathrm{m}^{2}$. This wide range of conductances resulted in changes in the somatic firing rate (Figure 6). In the case when we varied $g_{\mathrm{CaP}}$ the firing rate approached zero when $g_{\mathrm{CaP}}<40 \mathrm{~S} / \mathrm{m}^{2}$. Varying $g_{\mathrm{Kc}}$ resulted in an almost linear dependence of the firing rate with the firing rate at each conductance level less than $10 \%$ from the collective average (Figure 6B).

The information analysis of the conductance manipulations shown in Figure 6 result in that the entropy of $I_{\mathrm{CaP}}$ as a function of $g_{\mathrm{CaP}}$ decreases as $g_{\mathrm{CaP}}$ increased (Figure 7A left). This dependence is robust to changes in the value of the firing rate (Figure 6A). The conditional entropy between $I_{\mathrm{CaP}}$ and $I_{\mathrm{Glu}}$ had a slightly different dependence of $g_{\mathrm{CaP}}$ (center). The combination of entropy and conditional entropy resulted in a relatively constant value
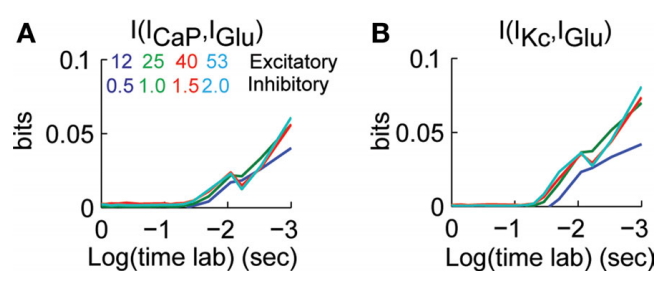

FIGURE 5 | Dependence of mutual information to previous activity. (A) III $\left.I_{\mathrm{CaP}}(t), I_{\mathrm{Glu}}(t-\Delta t)\right]$ for $\Delta t$ between $0-1 \mathrm{~s}$. (B) $\left.I I I_{\mathrm{Kc}}(t), I_{\mathrm{Glu}}(t-\Delta t)\right]$ for $\Delta t$ between $0-1 \mathrm{~s}$. The different traces correspond to different combinations of synaptic activity. Mutual information was bias corrected using the Panzeri and Treves method.
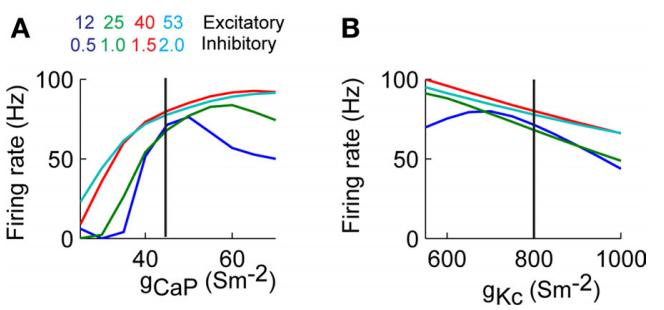

FIGURE 6 | Somatic firing rate varies as a function of dendritic excitability. (A) Firing rate as a function of homogenously changing the conductance of $\mathrm{CaP}\left(g_{\mathrm{CaP}}\right)$. (B) Similar to A changing the conductance of $\mathrm{Kc}$ $\left(g_{\mathrm{Kc}}\right)$. The vertical lines correspond to the values of the control simulation. 


\section{Entropy $\quad$ Conditional Entropy $\quad$ Mutual Information}
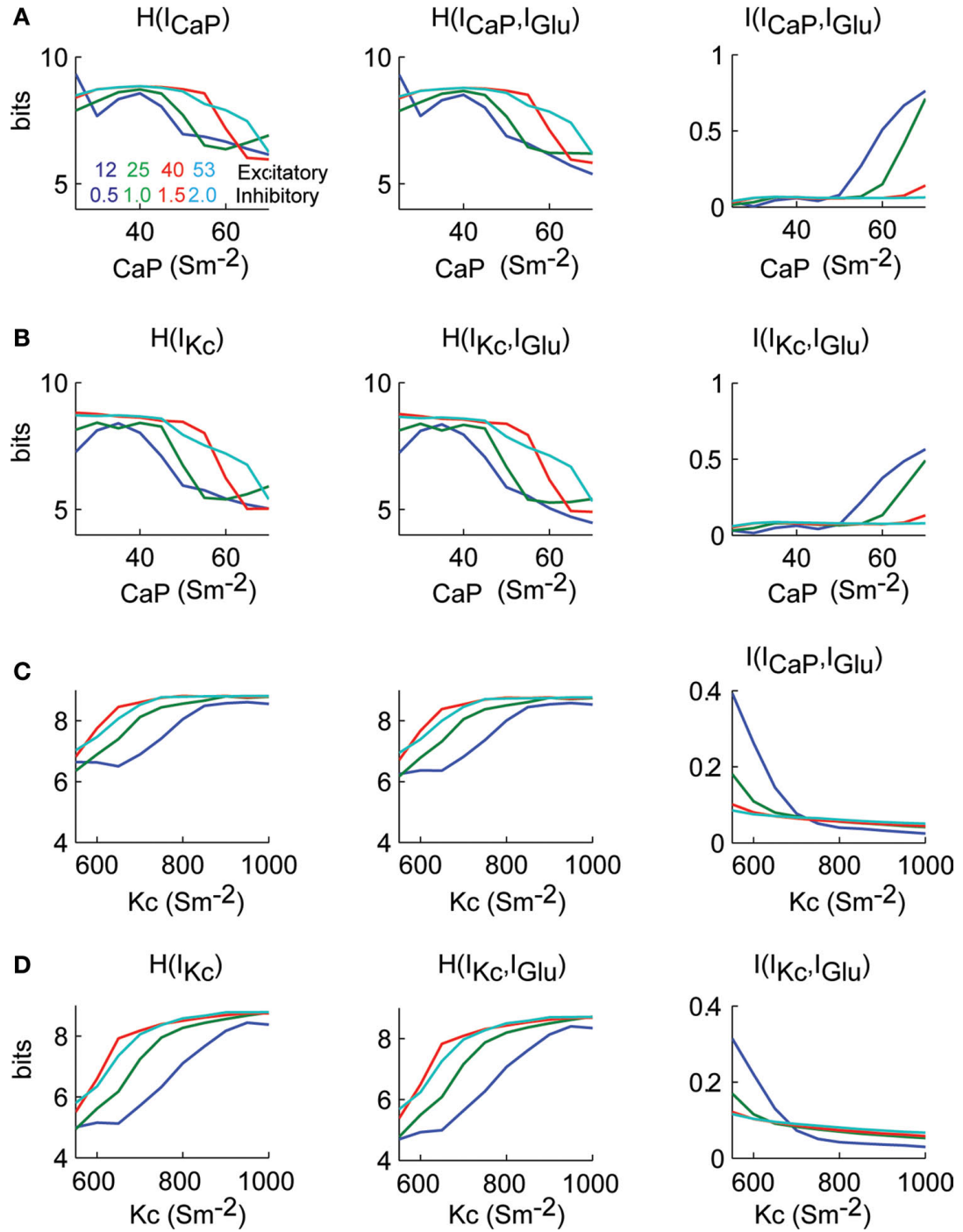

FIGURE 7 | Excitatory synaptic current information content in dendritic currents as a function of dendritic excitability. (A) Calculations $H\left(I_{\mathrm{CaP}}\right), H\left(I_{\mathrm{CaP}} \mid I_{G \mid \mathrm{u}}\right)$ and

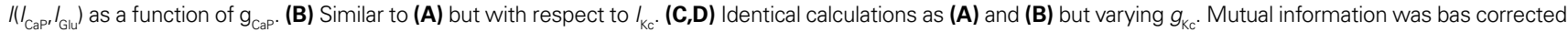
using the Panzeri and Treves method.

of the mutual information that then increased as a function of background activity for increasing values of $g_{\mathrm{CaP}}$ (right). We repeated the same analysis to calculate information transmission of $I_{\mathrm{Glu}}$ through $I_{\mathrm{Kc}}$ as a function of $g_{\mathrm{CaP}}$ (Figure 7B). The functional dependence of the mutual information plots shown in Figures 7A,B showed a similar dependence. Note that the mutual information did not reflect the firing rate behavior. Similarly, we calculated mutual information from $I_{\mathrm{Glu}}$ to $I_{\mathrm{CaP}}$ and $I_{\mathrm{Kc}}$ as a function of $g_{\mathrm{Kc}}$ (Figures 7C,D). In these cases the mutual information increases as the $g_{\mathrm{Kc}}$ decreased, and the firing rate increased. This is due to the lower inhibitory effect of Kc over CaP. In practically all cases the ratio $\mathrm{N} / \mathrm{m}$ was larger than 1 (Figures S3 and S4 in Supplementary Material).
We further quantified the effect of time lags and dendritic excitability on information processing of $I_{\text {Glu }}$ in the dendrite. We calculated the mutual information of $I_{\mathrm{CaP}}$ with $I_{\mathrm{Glu}}$ as a function of $g_{\mathrm{CaP}}$ and time lags up to $1 \mathrm{~s}$ (Figure 8A), for all levels of background activity. This analysis shows that for high values of $g_{\mathrm{CaP}}$ and low levels of background activity mutual information is maximized (Figure 8A right). The decay of the mutual information increases as a function of the value of $g_{\mathrm{CaP}}$. A similar set of results is obtained when calculating mutual information content of $I_{\mathrm{Glu}}-I_{\mathrm{Kc}}$ (Figure 8B). However, in this case, the effect of the background level of stimulation is not as strong as in $I_{\mathrm{CaP}}$. A more subtle influence is shown by a similar analysis of the effects on the mutual information of $I_{\mathrm{Glu}}$ with $I_{\mathrm{CaP}}$ and $I_{\mathrm{Kc}}$ while varying $g_{\mathrm{Kc}}$ (Figures $\mathbf{8 C}, \mathbf{D}$, respectively). Overall, this 
A

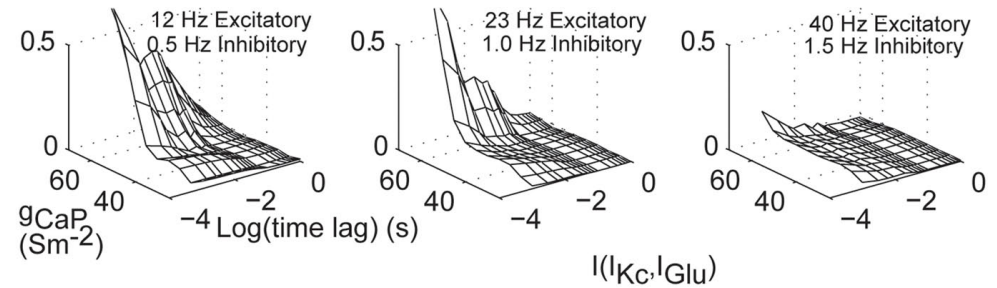

B
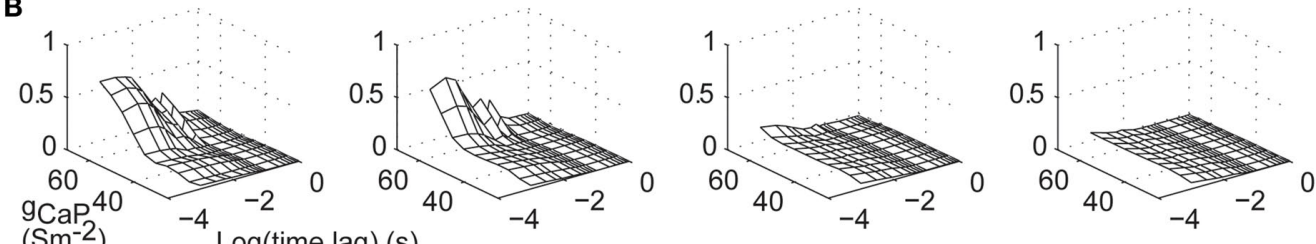

C

$\mathrm{I}\left(\mathrm{I}_{\mathrm{CaP}}, \mathrm{I}_{\mathrm{Glu}}\right)$
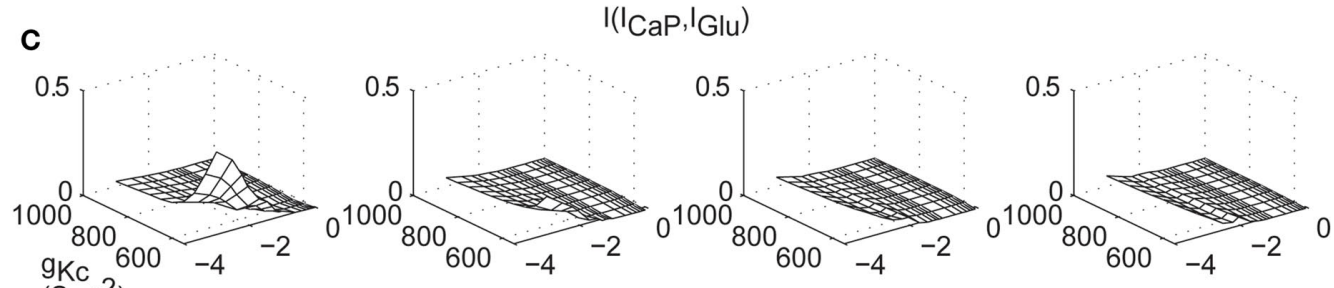

$\left(\mathrm{Sm}^{-2}\right)$

Log(time lag) (s)

$\mathrm{I}\left(\mathrm{I}_{\mathrm{Kc}},{ }^{\mathrm{I}} \mathrm{Glu}\right)$

D
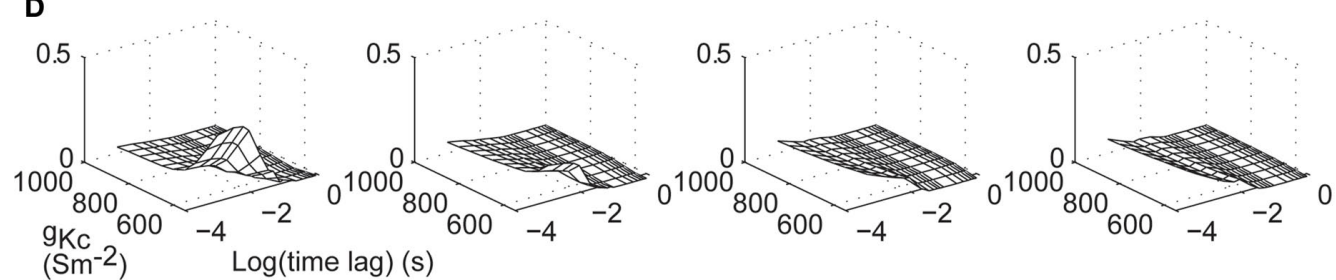

FIGURE 8 | Excitatory synaptic current information content in dendritic currents as a function of dendritic excitability and time lags. (A) $/\left[I_{\mathrm{CaP}}(t), I_{\mathrm{Glu}}\right.$ $(t-\Delta t)$ ] for $\Delta t$ from $0-1 \mathrm{~s}$ and varying $g_{\mathrm{CaP}}$. (B) As in $(\mathbf{A})$ for $I_{\mathrm{Kc}}$. (C,D) Identical calculations as in (A-B) but varying $g_{\mathrm{Kc}}$. The different panels correspond to different combinations of synaptic activity. Mutual information was bias corrected using the Panzeri and Treves method. analysis shows that the window of dendritic integration is directly modulated not only by the excitability provided by active conductances but also by the level of synaptic stimulation, especially in the case of $I_{\mathrm{K} c}$. Since both of these properties are dynamic and plastic, they allow the cell to regulate synaptic information processing.

\section{DISCUSSION}

Using a Purkinje cell model we presented a statistical analysis that shows that most of the information encoded in the background excitatory input is carried by the $\mathrm{CaP}$ and Kc currents. We have shown that the excitability of the dendritic tree represented by the density of active dendritic conductances modulates the information content of the total excitatory input in conjunction with the amount of synaptic activity. Consequently, the time window of integration of synaptic activity is in itself dependent on the level of synaptic activity which is not necessarily reflected in the spiking activity at the soma. This dynamical range of information processing consolidates the view that Purkinje cell dendrites processes synaptic activity locally before delivering it to the soma and provide a more sophisticated function than to compensate for the electrotonic decay of synaptic signals (Segev and London, 2000; Bekkers and Hausser, 2007).

\section{INFORMATION CHANNELS IN DENDRITES}

Neuronal response to synaptic activity is an analog-to-digital transformation that requires the integration and processing of a large number of synaptic inputs distributed across a dendrite (Segev and London, 2000). Traditionally, information theory has been applied to determine how much of the input stimulus can be reconstructed from observing the spiking activity of a neuron (Rieke, 1997). Under this approach, the neuron is lumped into a point source function in which dendritic processing is lost (Harris and Stark, 1972; Sherry and Klemm, 1980; Yamada et al., 1996; Borst and Theunissen, 1999; Levy and Baxter, 2002; Schultz et al., 2009). In this study we refined the use of mutual information and linked it to a biophysical property of dendrites. In this framework, each conductance is treated as an information channel that communicates 
and interacts with other information channels in the dendrite. We found that the mutual information between excitatory synaptic input and dendritic currents varies as a function of dendritic excitability and the level of synaptic activity.

Although the activity of dendritic currents is dependent on $I_{\mathrm{Glu}}$ stimulation in the past our analysis shows strong mutual information at lag 0 . This 'background' information stems from the overall level of activity in the dendrite as also shown by the cross-correlation analysis. These results are dependent on the properties of the stimulus that were meant to replicate the continuous activity arriving from parallel fibers and inhibitory synapses. This stimulation resulted in an overall level of activity of dendritic channels that is reflected at zero lag time. However, the second peak shown in the cross-correlation and mutual information is more indicative of the internal dynamics of the dendritic currents and the information content of the instantaneous value of $I_{\mathrm{Glu}}$. The differential encoding of synaptic activity underscores the modulatory property of the parallel fiber system on Purkinje cell activity (Santamaria et al., 2007; Walter et al., 2009).

Purkinje cells also receive synaptic activity from the ascending segment part of the granule cell axon and climbing fibers (Llinas, 1982). Both of these synaptic inputs deliver a more instantaneous stimulus lasting a few milliseconds. Under such types of stimuli and in the absence of background parallel fiber stimulation the instantaneous mutual information would be zero and would increase to reach a maximum, possibly at $20 \mathrm{~ms}$, as indicated by our crosscorrelation and mutual information analyses. Thus, mutual information from different systems could be encoded differently in the Purkinje cell dendritic tree.

The time window in which we define our stimulus affects the value of the mutual information. For example, our window of analysis ( $1 \mathrm{~ms}$ ) would be too slow to study information at the some where fast channels are present. However, other types of cells could have fast dendritic channels (Regehr and Tank, 1992). Thus, caution should be taken when applying information theory to analyzing dendritic activity.

\section{USE OF MUTUAL INFORMATION TO TUNE BIOPHYSICAL MODELS AND EXPERIMENTAL DESIGN}

Bounding the kinetic and density parameters of dendritic current models has been difficult since present experimental techniques are limited in resolving the spatial distribution of dendritic conductances (Achard and De Schutter, 2008; Van Geit et al., 2008). We propose that by treating each dendritic conductance as an information channel it is possible to constrain the value and distribution of dendritic currents in encoding synaptic information. Although here we have presented data regarding encoding the total excitatory current, the same technique can be extended to total inhibitory, total synaptic current, or it can be subdivided into the different parts of the dendritic tree (primary, secondary, and tertiary dendrites). At a first iteration, traditional template matching algorithms could be used to fit a model to

\section{REFERENCES}

Achard, P., and De Schutter, E. (2008). Calcium, synaptic plasticity and intrinsic homeostasis in Purkinje neuron models. Front Comput Neurosci 2:8. doi: 10.3389/neuro.10.008.2008
Barnes, C. A., McNaughton, B. L., and O’Keefe, J. (1983). Loss of place specificity in hippocampal complex spike cells of senescent rat. Neurobiol. Aging 4, 113-119.

Bekkers, J. M., and Hausser, M. (2007). Targeted dendrotomy reveals active

data. These algorithms change the density of dendritic conductances (Achard and De Schutter, 2008). Then models could be classified due to their value of mutual information between the stimulus and each dendritic current. Using this approach we could test the hypothesis that by matching the spiking activity at the soma the information content of the synaptic activity in dendritic currents is maximized.

The simulation analysis presented in this work can be used to design experiments based on dynamic clamp technique (Sharp et al., 1993; Kreiner and Jaeger, 2004). A challenging experiment would consist in patching a Purkinje cell while blocking spiking activity with tetrodotoxin. The pipette could deliver artificial synaptic conductances while monitoring the resulting current in the soma. The mutual information between the synaptic input and the total dendritic current value could be calculated from this experiment. Pharmacological manipulation blocking dendritic calcium or calcium activated potassium channels in the dendrite would then uncover the contribution to information processing. Other similar experiments could be to electrically or photochemically stimulate a group of synapses while recording at the soma (Korkotian and Segal, 2006) or to use the recently developed targeted dendrotomy technique (Bekkers and Hausser, 2007).

\section{BIOPHYSICAL INTERPRETATION}

Dendritic excitability, in Purkinje and other cell types, is the consequence of the different concentration and distribution of active conductances over a dendritic tree (Gunay et al., 2008; Komendantov and Ascoli, 2009). Neuronal stimulation and development change the excitability of the cell and could be a mechanism for storing memories (Womack and Khodakhah, 2003; Kim and Linden, 2007). Similarly, excitability is affected by aging and illnesses that could reduce the information capacity of the dendrite (Landfield and Pitler, 1984; Chan et al., 2007; Kabaso et al., 2009). It is well established that hippocampal pyramidal cells firing rate remains constant through aging (Barnes et al., 1983; Wilson et al., 2005) but not in Purkinje cells (Rogers et al., 1980), however the contribution of dendritic excitability and afferent stimulation on these changes is not well understood. In any case, our analyses have shown that changes in $g_{\mathrm{CaP}}$ or $g_{\mathrm{Kc}}$ can regulate the amount of information encoded and the time synaptic activity affects neuronal activity. As the hyperpolarizing Kc current decreases, the information contained in $I_{\mathrm{CaP}}$ and $I_{\mathrm{Kc}}$ increases potentially allowing more information to be transmitted by the neuron (Steuber et al., 2007).

\section{ACKNOWLEDGMENTS}

UTSA-TRAC and NSF HRD-0932339.

\section{SUPPLEMENTARY MATERIAL}

The Supplementary Material for this article can be found online at http://www.frontiersin.org/computationalneuroscience/ paper/10.3389/fncom.2010.00006/

and passive contributions of the dendritic tree to synaptic integration and neuronal output. Proc. Natl. Acad. Sci. U.S.A. 104, 11447-11452.

Borst, A., and Theunissen, F. E. (1999) Information theory and neural coding. Nat. Neurosci. 2, 947-957.
Bower, J. M. (2002). The organization of cerebellar cortical circuitry revisited: implications for function. Ann. N. Y. Acad. Sci. 978, 135-155.

Chan, C. S., Guzman, J. N., Ilijic, E. Mercer, J. N., Rick, C., Tkatch, T., Meredith, G. E., and Surmeier, D. J. 
(2007)./'Rejuvenation/' protects neurons in mouse models of Parkinson/'s disease. Nature 447, 1081-1086.

De Schutter, E., and Bower, J. M. (1994a). Simulated responses of cerebellar Purkinje cells are independent of the dendritic location of granule cell synaptic inputs. Proc. Natl. Acad. Sci. U.S.A. 91, 4736-4740.

De Schutter, E., and Bower, J. M. (1994b). An active membrane model of the cerebellar Purkinje cell II. Simulation of synaptic responses. J. Neurophysiol. 71, 401-419.

De Schutter, E., and Bower, J. M. (1994c). An active membrane model of the cerebellar Purkinje cell. I. Simulation of current clamps in slice. J. Neurophysiol. 71, 375-400.

De Schutter, E., and Steuber, V. (2009). Patterns and pauses in Purkinje cell simple spike trains: experiments, modeling and theory. Neuroscience.162, 816-826.

Foffani, G., and Priori, A. (2007). Information theory, single neurons and gamma oscillations in the human subthalamic nucleus. Exp. Neurol. 205, 292-293.

French, C. R., Sah, P., Buckett, K. J., and Gage, P. W. (1990). A voltagedependent persistent sodium current in mammalian hippocampal neurons. J. Gen. Physiol. 95, 1139-1157.

Gollo, L. L., Kinouchi, O., and Copelli, M. (2009). Active dendrites enhance neuronal dynamic range. PLoS Comput. Biol. 5, e1000402. doi:10.1371/journal. pcbi. 1000402 .

Gruol, D. L., Jacquin, T., and Yool, A. J. (1991). Single-channel K+ currents recorded from the somatic and dencritic regions of cerebellar Purkinje neurons in culture. J. Neurosci. 11, 1002-1015.

Gunay, C., Edgerton, J. R., and Jaeger, D. (2008). Channel density distributions explain spiking variability in the globus pallidus: a combined physiology and computer simulation database approach. J. Neurosci. 28, 7476-7491.

Gundappa-Sulur, G., De Schutter, E., and Bower,J.M. (1999). Ascending granule cell axon: an important component of cerebellar cortical circuitry. J. Comp. Neurol. 408, 580-596.

Harris, D. A., and Stark, L. (1972). Information theoretical analysis of a crayfish behavioral system. Brain Res. 36, 425-430.

Hirano, T., and Hagiwara, S. (1989). Kinetics and distribution of voltagegated $\mathrm{Ca}, \mathrm{Na}$ and $\mathrm{K}$ channels on the somata of rat cerebellar Purkinje cells. Pflugers Arch. Eur. J. Physiol. 413, 463-469.

Hirst, G. D., and McLachlan, E. M. (1986). Development of dendritic calcium currents in ganglion cells of the rat lower lumbar sympathetic chain. J. Physiol. (Lond.) 377, 349-368.

Hockberger, P.E., Tseng, H.Y., and Connor, J. A. (1989). Fura-2 measurements of cultured rat Purkinje neurons show dendritic localization of $\mathrm{Ca} 2+$ influx. J. Neurosci. 9, 2272-2284.

Huguenard, J. R., Hamill, O. P., and Prince, D.A. (1989). Sodium channels in dendrites of rat cortical pyramidal neurons. Proc. Natl. Acad. Sci. U.S.A. 86, 2473-2477.

Jaeger, D., and Bower, J. M. (1994). Prolonged responses in rat cerebellar Purkinje cells following activation of the granule cell layer: an intracellular in vitro and in vivo investigation. Exp. Brain Res. 100, 200-214.

Jaeger, D., and Bower, J. M. (1999). Synaptic control of spiking in cerebellar Purkinje cells: dynamic current clamp based on model conductances. J. Neurosci. 19, 6090-6101.

Jaeger, D., De Schutter, E., and Bower, J. M. (1997). The role of synaptic and voltage-gated currents in the control of Purkinje cell spiking: a modeling study. J. Neurosci. 17, 91-106.

Kabaso, D., Coskren, P. J., Henry, B. I., Hof, P. R., and Wearne, S. L. (2009). The electrotonic structure of pyramidal neurons contributing to prefrontal cortical circuits in macaque monkeys is significantly altered in aging. Cereb. Cortex. 19, 2248-2268.

Kaneda, M., Wakamori, M., Ito, C., and Akaike, N. (1990). Low-threshold calcium current in isolated Purkinje cell bodies of rat cerebellum. J. Neurophysiol. 63, 1046-1051.

Kennel, M. B., Shlens, J., Abarbanel, H. D., and Chichilnisky, E. J. (2005). Estimating entropy rates with Bayesian confidence intervals. Neural. Comput. 17, 1531-1576.

Kim, S. J., and Linden, D. J. (2007). Ubiquitous plasticity and memory storage. Neuron 56, 582-592.

Komendantov, A. O., and Ascoli, G. A. (2009). Dendritic excitability and neuronal morphology as determinants of synaptic efficacy. J. Neurophysiol. 101, 1847-1866.

Korkotian, E., and Segal, M. (2006). Spatially confined diffusion of calcium in dendrites of hippocampal neurons revealed by flash photolysis of caged calcium. Cell Calcium. 40, 441-449.

Kreiner, L., and Jaeger, D. (2004). Synaptic shunting by a baseline of synaptic conductances modulates responses to inhibitory input volleys in cerebellar Purkinje cells. Cerebellum 3, 112-125.

Landfield, P., and Pitler, T. (1984). Prolonged Ca2+-dependent afterhyperpolarizations in hippocampal neurons of aged rats. Science 226, 1089-1092.

Latorre, R., OBerhauser, A., Labarca, P., and Alvarez, O. (1989). Varieties of calcium-activated potassium channels. Annu. Rev. Physiol. 51, 385-399.

Levy, W. B., and Baxter, R. A. (2002). Energy-efficient neuronal computation via quantal synaptic failures. $J$. Neurosci. 22, 4746-4755.

Llinas, R. (1982). "Radial connectivity in the cerebellar cortex: a novel view regarding the functional organization of the molecular layer," in The cerebellum: new vistas, eds S. Plalay and C. Chan-Palay (New York: Elsevier), 189-184.

Llinas, R. R., Sugimori, M., and Cherksey, B. (1989). Voltage-dependent calcium conductances in mammalian neurons. The P channel. Ann. N.Y. Acad. Sci. 560, 103-111.

Llinas, R., and Yarom, Y. (1981) Electrophysiology of mammalian inferior olivary neurones in vitro. Different types of voltage-dependent ionic conductances. J. Physiol. 315, 549-567.

London, M., Schreibman, A., Hausser, M., Larkum, M. E., and Segev, I. (2002). The information efficacy of a synapse. Nat. Neurosci. 5, 332-340.

Maffezzoli, A., Signorini, M. G., Gullo, F., and Wanke, E. (2008). Modeling spiking activity of in vitro neuronal networks through non linear methods. Conf. Proc. IEEE Eng. Med. Biol. Soc. 2008, 42-45.

Magri, C., Whittingstall, K., Singh, V., Logothetis, N. K., and Panzeri, S. (2009). A toolbox for the fast information analysis of multiple-site LFP, EEG and spike train recordings. $B M C$ Neurosci. 10, 81.

Marr, D. (1969). A theory of cerebellar cortex. J. Physiol. (Lond.) 202, 437-470.

Masukawa, L. M., Hansen, A. J., and Shepherd, G. (1991). Distribution of single-channel conductances in cultured rat hippocampal neurons. Cell. Mol. Neurobiol. 11, 231-243.

Migliore, M., and Shepherd, G. M. (2002). Emerging rules for the distributions of active dendritic conductances. Nat. Rev. Neurosci. 3, 362-370.

Nemenman, I., Bialek, W., and de Ruyter van Steveninck, R. (2004). Entropy and information in neural spike trains: progress on the sampling problem. Phys. Rev. E Stat. Nonlin. Soft Matter Phys. 69, 056111.

Paninski, L., Pillow, J. W., and Simoncelli, E.P. (2004). Maximum likelihood estimation of a stochastic integrate-andfire neural encoding model. Neural Comput. 16, 2533-2561.

Panzeri, S., Senatore, R., Montemurro, M. A., and Petersen, R. S. (2007). Correcting for the sampling bias problem in spike train information measures. J. Neurophysiol. 98, 1064-1072.

Pellionisz, A., and Szentagothai, J. (1973). Dynamic single unit simulation of a realistic cerebellar network model. Brain Res. 49, 83-99.

Pellionisz, A., and Szentagothai, J. (1974). Dynamic single unit simulation of a realistic cerebellar network model. II. Purkinje cell activity within the basic circuit and modified by inhibitory systems. Brain Res. 68, 19-40.

Poirazi, P., and Mel, B. W. (2001). Impact of active dendrites and structural plasticity on the memory capacity of neural tissue. Neuron 29, 779-796.

Quian Quiroga, R., and Panzeri, S. (2009). Extracting information from neuronal populations: information theory and decoding approaches. Nat. Rev. Neurosci. 10, 173-185.

Rall, W. (1962a). Electrophysiology of a dendritic neuron model. Biophys. J. 2, 145-167.

Rall, W. (1962b). Theory of physiological properties of dendrites. Ann. N. Y Acad. Sci. 96, 1071-1092.

Regehr, W. G., and Tank, D. W. (1992). Calcium concentration dynamics produced by synaptic activation of CA1 hippocampal pyramidal cells. $J$. Neurosci. 12, 4202-4223.

Rieke, F. (1997). Spikes: Exploring the Neural Code. Cambridge, MA: MIT Press.

Rogers, J., Silver, M. A., Shoemaker, W. J., and Bloom, F. E. (1980). Senescent changes in a neurobiological model system: cerebellar Purkinje cell electrophysiology and correlative anatomy. Neurobiol. Aging 1, 3-11.

Santamaria, F., and Bower, J. M. (2005). Background synaptic activity modulates the response of a modeled Purkinje cell to paired afferent input. J. Neurophysiol. 93, 237-250.

Santamaria, F., Jaeger, D., De Schutter, E., and Bower, J. M. (2002). Modulatory effects of parallel fiber and molecular layer interneuron synaptic activity on Purkinje cell responses to ascending segment input: a modeling study. J. Comput. Neurosci. 13, 217-235.

Santamaria, F., Tripp, P. G., and Bower, J. M. (2007). Feedforward inhibition controls the spread of granule cell-induced Purkinje cell activity in the cerebellar cortex. J. Neurophysiol. 97, 248-263.

Schultz, S. R., Kitamura, K., PostUiterweer, A., Krupic, J., and Hausser, M. (2009). Spatial pattern coding of sensory information by climbing fiber-evoked calcium signals in networks of neighboring cerebellar Purkinje cells. J. Neurosci. 29, 8005-8015.

Segev, I., and London, M. (1999). “A theoretical view of passive and active dendrites," in Dendrites, eds G. Stuart, S. Nelson and M. Hausser (Oxford: Oxford university press), 205-230.

Segev, I., and London, M. (2000). Untangling dendrites with quantitative models. Science 290, 744-750. 
Shannon, C. E. (1948). A mathematical theory of communication. Bell Syst. Tech. J. 27, 379-423, 623-656.

Shannon, C. E., and Weaver, W. (1949). The Mathematical Theory of Communication. Urbana: University of Illinois Press.

Sharp, A. A., Oneil, M. B., Abbott, L. F., and Marder, E. (1993). The dynamic clamp - artificial conductances in biological neurons. Trends Neurosci. 16, 389-394.

Sherry, C. J., and Klemm, W. R. (1980). The statistical relationship between the "entropy" of a neuronal signal and its variability. Int. J. Neurosci. 11, 109-113.

Spain, W. J., Schwindt, P. C., and Crill, W. E. (1987). Anomalous rectification in neurons from cat sensorimotor cortex in vitro. J. Neurophysiol. 57, 1555-1576.

Stemmler, M., and Koch, C. (1999). How voltage-dependent conductances can adapt to maximize the information encoded by neuronal firing rate. Nat. Neurosci. 2, 521-527.

Steuber, V., Mittmann, W., Hoebeek, F. E., Silver, R. A., De Zeeuw, C. I., Häusser,
M., and De Schutter, E. (2007). Cerebellar LTD and pattern recognition by Purkinje cells. Neuron 54, 121-136. Stuart, G. J., and Hausser, M. (2001). Dendritic coincidence detection of EPSPs and action potentials. Nat. Neurosci. 4, 63-71.

Suter, K. J., and Jaeger, D. (2004). Reliable control of spike rate and spike timing by rapid input transients in cerebellar stellate cells. Neuroscience 124, 305-317.

Van Geit, W., De Schutter, E., and Achard, P. (2008). Automated neuron model optimization techniques: a review. Biol. Cybern. 99, 241-251.

Vetter, P., Roth, A., and Hausser, M. (2001). Propagation of action potentials in dendrites depends on dendritic morphology. J. Neurophysiol. 85, 926-937.

Victor, J. D. (2000). Asymptotic bias in information estimates and the exponential (Bell) polynomials. Neural. Comput. 12, 2797-2804.

Walter, J. T., Dizon, M.-J., and Khodakhah, K. (2009). The functional equivalence of ascending and parallel fiber inputs in cerebellar computation. J. Neurosci. 29, 8462-8473.

Wilson, I. A., Ikonen, S., Gallagher, M., Eichenbaum, H., and Tanila, $\mathrm{H}$. (2005). Age-associated alterations of hippocampal place cells are subregion specific. J. Neurosci. 25, 6877-6886.

Womack, M. D., and Khodakhah, K. (2003). Somatic and dendritic smallconductance calcium-activated potassium channels regulate the output of cerebellar Purkinje neurons. J. Neurosci. 23, 2600-2607.

$\mathrm{Xu}, \mathrm{J}$., and Kang, J. (2005). The mechanisms and functions of activitydependent long-term potentiation of intrinsic excitability. Rev. Neurosci. 16, 311-323.

Yamada, S., Matsumoto, K., Nakashima, M., and Shiono, S. (1996). Information theoretic analysis of action potential trains. II. Analysis of correlation among $n$ neurons to deduce connection structure. J. Neurosci. Methods 66, 35-45.

Yamada, W. M., Koch, C., and Adams, P. R. (1989). "Multiple channels and calcium dynamics," in Methods in Neuronal Modeling: From Synapses to Networks, eds C. Koch and I. Segev (Cambridge: MIT Press), 97-133.

Conflict of Interest Statement: The authors declare that the research was conducted in the absence of any commercial or financial relationships that could be construed as a potential conflict of interest.

Received: 06 July 2009; paper pending published: 06 August 2009; accepted: 05 March 2010; published online: 30 March 2010.

Citation: Coop AD, Cornelis $H$ and Santamaria F (2010) Dendritic excitability modulates dendritic information processing in a Purkinje cell model. Front. Comput. Neurosci. 4:6. doi: 10.3389/fncom.2010.00006

Copyright (c) 2010 Coop, Cornelis and Santamaria. This is an open-access article subject to an exclusive license agreement between the authors and the Frontiers Research Foundation, which permits unrestricted use, distribution, and reproduction in any medium, provided the original authors and source are credited. 